\title{
New model of Electronic Health Record: Macedonian case study
}

\author{
Goce Gavrilov $^{1}$, Vladimir Trajkovik ${ }^{2}$ \\ ${ }^{1}$ Health Insurance Fund of Macedonia, ul. Macedonia 5, Skopje, Macedonia \\ gavrilovgoce@yahoo.com \\ ${ }^{2}$ Faculty of computer science and Engineering, "Ss. Cyril and Methodius" University, \\ Rugjer Boskovikj 16, Skopje, Macedonia \\ trvlado@finki.ukim.mk
}

\begin{abstract}
Electronic Health Record (EHR) systems are core applications in any e-Health environment and represent basic services for healthcare information technology platforms. Many EHR introduction projects ran into trouble because of the inappropriate model and concept of implementation, according to the requirements and standards, as well as the lack of the acceptance of the EHR related services among physicians. The aim of this paper is to give a brief overview of the current situation of citizens' healthcare data and need for the nationwide EHR with focus on R. Macedonia. We are making an analysis of the best practices of EHR's implementation and introduction in many countries in the world. We use these positive and useful experiences in the Macedonian case of EHR's introduction and propose a new collaborative model of EHR for healthcare organizations, adapted to the situation and needs of the Macedonian healthcare information system.
\end{abstract}

Keywords: Electronic health record (EHR), e- services, healthcare, collaboration

\section{Introduction}

The development in the area of information and communication technologies and progress in medical sciences, together with related technologies, has led to a strong need for exchange health information across institutional borders. New information and communication technologies have the potential to revolutionize healthcare and health systems and contribute to their future sustainability. Many IT systems have been developed and implemented in attempts to improve healthcare protection. Some of these systems include simple electronic health records (EHR) or electronic medical records (EMR) that replace paper records for information storage and retrieval [1]. Walji et al. [2] recommend the usage of a multi method approach when testing the usability of health information technology. 
Health information technologies (HIT) represent one of the most promising ways to modernize and improve health care delivery. Using of HIT provides timely access to patient health information and evidence-based decision support thus reducing medical errors and enhance clinical decision making [3]. Electronic health record (EHR), electronic patient record (EPR), electronic medical record (EMR), Healthcare Information Systems and the exchange of healthcare data are essential components of the IT infrastructure in healthcare. The same are often crucial elements in providing the support for health care reforms.

EHR describes the concept of a comprehensive, institutional, and longitudinal collection of a patient's health and healthcare data [4]. According to the definition from ISO/TR 20514 standard [4] EHR is defined as: 'a repository of information regarding the health status of a subject of care in computer process able form, stored and transmitted securely, and accessible by multiple authorized users'. EHR has a standardized or commonly agreed logical model of information which is independent of EHR systems. The main purpose of EHR is the support of continuing, efficient and quality integrated healthcare.

EHR includes both medical and non-traditional health and lifestyle-related information, with the consumer as the focus around which the information is recorded. Different health care professionals and administrative staff are using the EHR: physicians, nurses, pharmacists, laboratory technicians and other healthcare employees.

EMR systems manage electronic health records, so they can be created, gathered, and managed by authorized healthcare providers within healthcare organizations. Data entered into the computer (e.g. X-ray, pathology and pharmacy data) which can be integrated into the record are called electronic medical record. In some situation, there may be paper back up. EMR may be characterized as a partial health record under the hat of a healthcare provider that holds a portion of the relevant health information about a person over their lifetime.

An EPR is a sub-type of an EHR, used in a specific hospital or healthcare organization. According to the [4] it's defined as 'a repository of patient data in digital form, stored and exchanged securely and accessible by multiple authorized users'.

EHRs differ from EMRs and PHRs most notably on the basis of the completeness of the information the record contains and the designated custodian of the information. A PHR is often described as a complete or partial health record under the custodian of a person(s) that holds all or a portion of the relevant health information about that person over their lifetime.

Interoperability in healthcare refers to the possibility of exchanging health data between two or more interconnected systems. The exchange information should be interpreted and understood in the same way for all the interconnected systems. To reduce costs and improve quality of healthcare, cooperation and collaboration of healthcare units from different locations is required.

The introduction of national platforms for managing of EHR can be treated as a new health information system. In this paper certain design and organizational aspects in Macedonian case of National EHR will be considered. This paper describes a new collaborative model of EHR for healthcare organizations, adapted to the needs of the Macedonian healthcare information system.

In the next section a brief description of the current situation of the EHR in Macedonia is given. Description of new collaborative model of EHR is presented in section Results. 
Discussion and challenges are presented in section Discussion, while last section concludes the paper.

\section{Current situation of EHR in Republic of Macedonia}

Health services in Macedonia, like most of European countries, are divided between primary, secondary and tertiary care. Primary care is health care provided by the physicians of general practice. Secondary care is medical attention provided by a specialist upon referral by a primary care physician, and tertiary care is provided by a team of specialists in a hospital or medical clinics.

In Macedonia, the basic conditions for an introduction of an EHR in different levels of healthcare has been provided. All citizens (insured people) are provided with a smart card called an electronic health card (EHC), which contains basic administrative information such as unique health number of the insurer, name, gender, etc. EHC is used for patient identification primarily by the healthcare institutions. The EHC also offers signaturefunctionality and can be upgraded to a citizens-card, which can be used for e-government applications or for private use [5].

In order to initiate the actual development of a healthcare information system (HIS) and EHR, in the past a document named 'Strategy for the Development of Macedonian Integrated Health Information System' [6] was prepared. The strategy contains a description of the current legal provisions, scientific, organizational, and functional environment, as well as the requirements for a basic HIS architecture and functionality.

With the reform of the primary healthcare, starting in 2006, the primary providers have been obliged to make regular reports to the HIF. This project was introduction in the idea for EHR. At this stage however, these records are visible and available only to the physicians, nurses and the HIF's staff for administrative purposes. Based on these efforts, Ministry of Health $(\mathrm{MoH})$ of Macedonia had attempt to implement EHR on a nationwide level. In the period from 2009 till 2011, an unsuccessful attempt for the implementation of EHR was made. The project failed because of the bad organization and the weak project management. There are many other examples in the literature review of 'failed' EHR implementations in healthcare [7].

This unsuccessful project has identified still-existing variances in the technical and IT capacities among healthcare providers, but moreover, lack of unified coding system for data entering as well as lack of standardized formats for data collection. So, several key initiatives for preparing the country for an integrated healthcare information system (such as the unified coding systems and electronic health records and cards) that would also be adaptable for the upcoming healthcare reforms (Diagnosis Related Groups (DRG)) were proposed. The unified coding system and the DRG in hospital care for the first time have been implemented in 2009 by HIF. Special software (DRG grouper) for keeping track of acute patients in hospitals was also implemented by HIF side. Following the initiatives of introduction of unified coding systems and uniformity in the operation of hospitals, HIF during the 2013 introduced a special software for collecting and processing data from daily clinics and specialized-consultative healthcare to secondary level.

Healthcare organizations in Macedonia use variety of IT applications and infrastructures which always need to be updated as a result of rapid growth in healthcare services. All of these healthcare organizations (on primary, secondary and tertiary level) 
collect and process medical data in separate IT subsystems. The diversification on how healthcare organizations maintain their operations, especially of patient's medical information, resulted into difficulties of accessing patient's data.

Apart from the EHC system, the health reformation law [8, 9], is one of the major formal steps towards an Introduction of electronic health record in Macedonia. The introduction of an EHR in Macedonia was explicitly defined as a goal opening legal opportunity to establish a National system of records in the healthcare field. The first module of this National System was national appointment system called 'My Appointment'.

Health Insurance Fund (HIF) of Macedonia has worked on several projects to modernize its information system. Regard this, the HIF implemented several electronic services (e-services) for insured people, companies and healthcare facilities [10,11]. HIF made a centralized registry for all health insured people in which administrative data, which are part of the EHR, are collected and processed. These data are available to all healthcare workers and health facilities through electronic services or special software interface (web services) [12].

Apart from the efforts taken by the government or health authorities, there are several initiatives and organizations establishing prototypes of EHRs or some parts of EHRs in Macedonia [13, 14]. These projects and initiatives closely cooperate with the concept of $\mathrm{MoH}$ initiatives, but do not share a more practical or scientific view on the EHR. EHR defined in these projects and initiatives contains partial data form EHR (not all data from nationwide EHR), EPR, EMR or Personal Health Record (PHR).

Introducing of EHR system on the nationwide, represents enormous change for the all stakeholders (physicians, nurses, and administrative staff in HIF, MoH, hospitals, and patients). Also, additional barriers for public acceptance of introduction of EHR are sensitive issues for privacy and security.

Today, in the Macedonian healthcare system, many data are still stored in human readable formats only. Although, data like laboratory results and medical findings are measured and calculated digitally, final reports are often printed and transmitted to the physician. In the modern healthcare systems, data are being stored and transmitted in computer's readable data formats.

On the other hand, although in the Macedonian health information system there are several separate information systems however the collaboration and data exchange between them is not satisfactory. HIF and the MoH in their IT system have developed specific web services for exchange of data, but they are not satisfactory and especially their mutual exchange and data synchronization. One system offers data exchange using digital signatures while the other is not, which is a problem.

Currently, there is a strong push in the Macedonian healthcare system for establishment and increase of the use of EHR in the delivery of health care. So, in this part of the paper we will proposed a model of EHR based on positive experiences in other countries, and structured according to the current situation in the Macedonian healthcare information systems. 


\section{New model of EHR for purposes of the Macedonian health information system}

\subsection{Model of EHR}

Different models of EHR are often discussed in literature in terms of degree of his centralization or distribution. According to the architecture of EHR, there is a separation of basic and universal model of EHR [15]. The basic model of EHR is built inside in one organization (hospital or clinic) and connected to all hospital/clinic IT systems as shown in Fig. 1. The basic EHR type is centralized and collects data from all operating healthcare systems in the hospitals, clinics or general practitioners. EPR and EMR can be considered as different kind of basic architecture model of EHR.

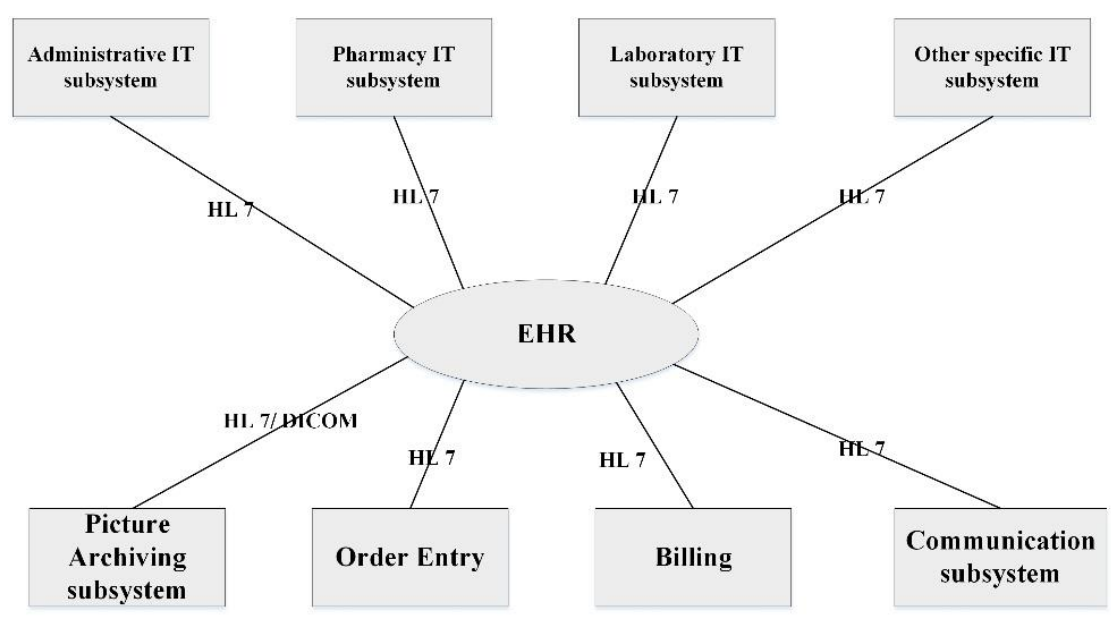

Fig. 1. Basic model of EHR

In centralized EHR patient data are collected and stored in a single repository or location. Individual healthcare providers or health professionals maintain the full details for individuals (patients) in their own EPR or EMR, which are subsets of the EHR. In Fig. 2 a centralized EHR model is shown. 


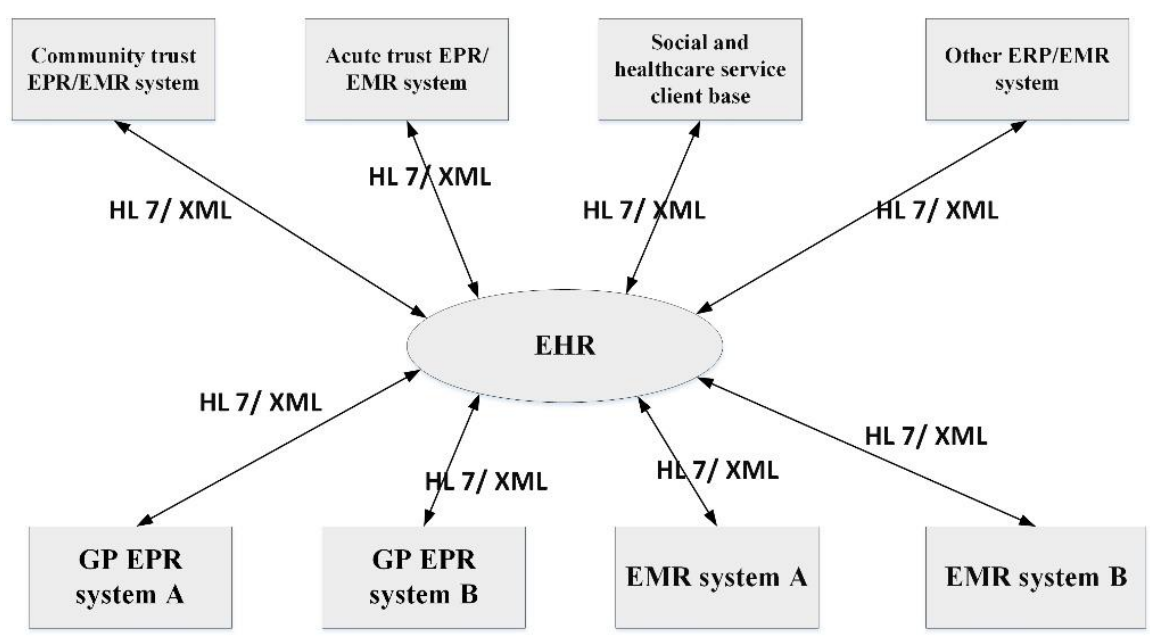

Fig. 2. Centralized model of HER

In the distributed model of EHR, there is not location that is considered as a primary repository of information. Instead, the EHR is physically distributed across several locations. Users create their own view or record by accessing the data from these locations. In this model of EHR (shown in Fig.3), each healthcare organization has its own EHR with its own data model and with its own terminology standards.

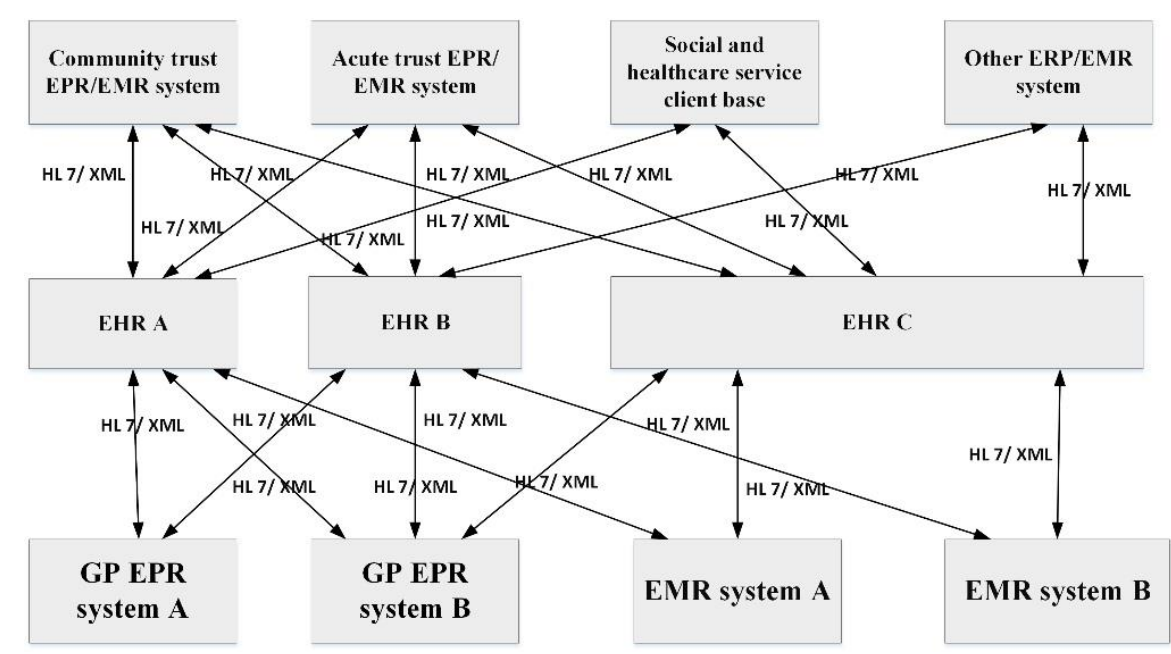

Fig. 3. Distributed model of EHR

In Macedonia, EHR's data are currently distributed in the different IT systems in private and public healthcare institutions. It is unclear how much of these data are relevant and enough for clinical research and which data elements are relevant for feasibility analyses. Structured data must be documented during patient care through the use of national or international sets or terminologies. Also, it is currently unclear what kind of 
data, besides data for reimbursement and administrative data, are available across Macedonian healthcare IT system.

Most of the laboratories have special laboratory information systems, so they have started structuring their data very early. But, there is still need for all laboratories to use standard terminologies and put their data with EHRs.

So, considering the current state of development of some parts of the EHR in the IT subsystems of all participants in the Macedonian health information system, centralized model of EHR would be most appropriate. This conclusion is based on the analysis of the administrative processes, the clinical workflows, data collection and reporting processes, Internet connectivity, staff member's computer skills and the required budget for implementation. Necessary budget for EHR's implementation, in public and private healthcare providers are key factor for implementation of EHR.

The development of communication links in the last few years, are a solid foundation for seamless access to information contained in EHR. Taking into account the current situation of medical and administrative data in Macedonian healthcare system, the choice of centralized EHR model is quite justified.

\subsection{Best practices of EHR's implementations and adequate technologies}

EHR's data, currently distributed in the different IT systems in private and public healthcare institutions, are in the quasi form of EHR, EMR and EPR. They are not defined and developed with specific standards. The data exchange between IT systems designed in this way, is very difficult. Hence, the introduction of standards in the design of the new model of EHR is need and should be taken into account in the design of new Macedonian model of EHR.

There are different health informatics standards that are currently used to define domain models of EHR: ISO HL7 21731 'Health informatics - HL7 version 3 Reference Information Model' (RIM) [16], ISO EN 13606 'Health Informatics - EHR communication' [17], Open - EHR Reference Model [18], etc. On the other hand, International Statistical Classification of Diseases 10th revision (ICD10) is used in most European EHRs.

Design of health information systems, especially their underlying business and informational models which describe basic concepts, business and relation networks, have to be based on standards. Lopez and Blobel present a method and the necessary tool for reusing standard of healthcare information models, for supporting the development of semantically interoperable systems and components [19].

The results of the Häyrinen et al. [1] literature reviews show that the challenge for ongoing national EHR projects, in the most of the countries, is to take into account all the different types of EHRs and the needs and requirements of different healthcare professionals and consumers in the development of EHRs. Additional challenge is the use of international terminologies and sets in order to achieve semantic interoperability.

We need to use these findings in the design of the Macedonian model of EHR. The data which are stored in EHR, are divided in two sections: administrative and medical content. The administrative content includes:

- Identification data - patient's complete name, medical record number, address, Mother's maiden name; and 
- Lifestyle indicators - education level, profession, allergies, chronic illnesses, marital status, food, preferences, smoking and alcohol consumption.

The data from medical content includes: Symptoms, physical examination results; Drugs prescribed, inpatient history; and Lab reports - pathology/radiology and other medical reports.

The EHR contains valuable information entered by physicians and clinicians. Besides its immediate clinical use at the point of care, the EHR provides rich data that can be analyzed and mined for clinical research (when EHR is treated as a repository of medical information across many patients).

Patient notes, in particular, convey an abundance of information about the patient's treatments and medical history, signs and symptoms, which often are not nested in the structured part of the EHR. Notes' information can be found in the form of narrative and semi-structured format through lists or templates with free-text fields [20].

Health Information Exchanges (HIE) is complex as the healthcare landscape is a complex network of varied stakeholders. At the center of this healthcare network is the patient. As the patient moves through the healthcare labyrinth, each stakeholder captures a slice of patient data or records a healthcare transaction pertaining to that patient. According to Metzger et al. [21], the interoperability of health information system is defined as: 'the capability of heterogeneous systems to interchange data in a way that the data from one can be recognized, interpreted, used and processed by other systems.'

Many countries have implemented a network of interoperable EHR. Among these countries is Canada with its so called 'Canada Health Infoway' [22]. According to Canadian model of interoperability, EHR solutions link clinics, hospitals, pharmacies and other healthcare providers. The vision of Canada Health Infoway is to provide a secure and private EHR lifetime record of health and healthcare history and care within the healthcare system, available to authorized healthcare providers, medical staff and individuals.

Following the Canadian 'Health Infoway', we can define interoperability infrastructure for the Macedonian case of EHR. In our case EHR should be host on backend of central healthcare information system. The main question is how these services will be exposed to the client consumers (primary, secondary and tertiary healthcare providers, and patients)?

According to Lomotey and Deters [23], two types of architectural designs from Web services have been embraced in the medical system design: SOA (Service-oriented Architecture) and ROA (Resource Oriented Architecture). SOA is an architectural style whose goal is to achieve loose coupling among interacting services. It has become an extremely popular paradigm. An enterprise service bus (ESB) is one of the main technologies that enable implementation of service-oriented architecture (SOA). As most appropriate, in our cases we use SOA.

Cloud computing technology offers cost-effective services (including simple data management and storage, cheap computer resources) and features (portability, reliability, scalability, and elasticity). It can be used as platform to implement EHR systems because it offers great potential for quick access to healthcare information and other information concerning health.

EHR can serve for multiple purposes including medical communication, legal documentation, financial transaction, research and analytics.

Electronic signatures attached to entries in EHRs have different logical and legal meanings for different users in the healthcare system. Some of these are stamps from 
historic paper formats that require reconsideration. Traditionally accepted functions of signatures, such as identity verification, consent, authorization and non-repudiation can become unclear in the context of computer-based workflow processes that incorporate functions like logins, auto-fill and audit trails.

The digital signature is a very useful mechanism that provides authenticity mechanisms in complex IT systems. With this security mechanism, the authenticity of the digital record is guaranteed and it will be sufficiently to deploy this kind of system in the Healthcare Cloud in order to avoid false data transactions. The digital signature gives the receiver the reassurance that a message or data are encrypted and protected.

Besides the previously mentioned positive experiences of implementation of some parts of EHR in European countries and implementation of HIS for the purposes of this paper we will use positive experiences of France, United States, United Kingdom, India [24], Lithuania [25], and results from 'failed' EHR implementations in healthcare [7]

\subsection{New model of EHR for Republic of Macedonia}

In this new model that we are proposing, EHRs are stored in the cloud, and can be accessed through a web portal or background web services (see Fig. 4). Owners of EHRs are responsible for generating access policies based on the attributes of authorized healthcare providers, encrypting EHRs and uploading encrypted EHRs, digital signing of all request for getting data and digital signing of response into the cloud. EHRs is organized into a hierarchical data structure, which give possibilities to share different parts of the EHR making our model more flexible.

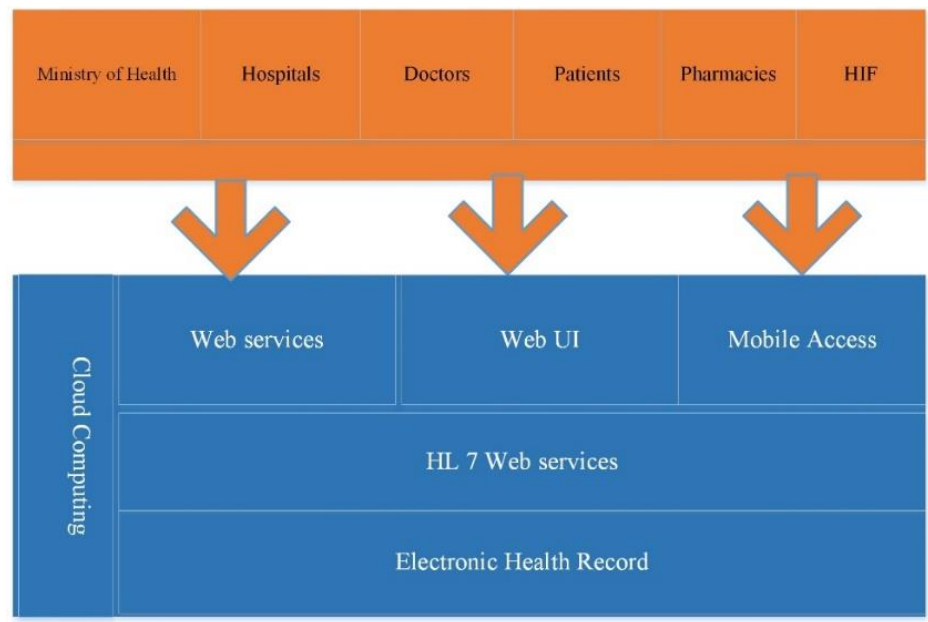

Fig. 4. New model of EHR

One of the access to the model of EHR is via web services. Direct call to web services can be used by the organizations that already have applications related to the health record and need to integrate those systems with the cloud based EHR. Access to the EHR through the web application can be used by healthcare organizations and providers that do not have EHR's application. The new EHR can be accessed through mobile application from 
smart devices. This is very convenient for the physicians and patients because they can use the application anytime and anywhere through their mobile devices.

For the data exchange between the entities involved in ours new model of EHR, we proposed the use of Health Level Seven International (HL7). Detail architecture of the proposed model of EHR is shown on Fig.5. The architecture consists of the following main parts:

- Interface access layer;

- Application layer; and

- Data management layer.

The Interface access layer represents the entry point to the EHR. It controls the access to the health data contained within the EHR. Interface access layer consists of an Identity management module and Access control module. Access control module provides access to all the actors authorized to access the data: patients/citizens, health professionals and administrative staff.

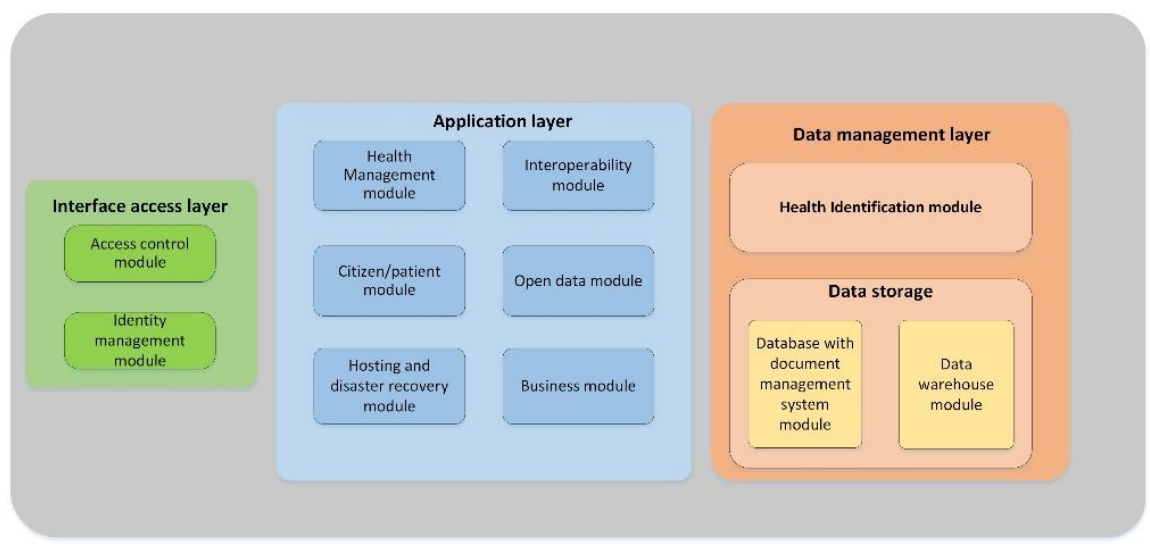

Fig. 5. Architecture of EHR

The main role of the Application layer is to implement all the user interfaces for interacting with the EHR's data. It has the following modules: Health management module, Citizen/patient module, Hosting and disaster recovery module, Interoperability module, Open data module and Business module.

Health management module suppors all the healthcare activities performed on EHR's by the authorized users. Citizen/patient module is a web based portal solution for the navigation through the patient's data. Whereas Interoperability module integrates the proposed EHR system with all external EHR subsystems, EMR, EPR. Hosting and disaster recovery module is for supporting and interacting with the cloud computing platform. Open data module is responsible for open data exchange with external users while Business module defines business rules and content data of EHR.

Data management layer have two system modules: Health Identification module and Data storage module.

The Health Identification module represents the central place for controlling the access to the sensitive medical information. It further consists of three submodules: Access manager submodule, Index Registry submodule and Event Manager submodule. Access manager submodule allows the citizen/patient to control the access to own data. Index 
Registry module allows EHR system to dialogue with a similar systems and to exchange data and resources. Event Manager submodule allows the EHR to track the creation of new data in the central database and to allocate it properly to the owner. This submodule brings the inheritance of all the default settings and access control.

The Data storage module consists of two submodules: Database with document management system submodule and data warehouse submodule. The database with document management system is a submodule specifically oriented to manage a vast variety of data collected within the EHR, including raw data, formatted documents, image files and so on. It represents an interface between data and the front-end application. The data warehouse submodule is an interface between the real EHR and the Database system that collect and manage the real data.

\section{Discussion}

Cloud computing can offer patients access to their medical data anytime and anywhere in the world. The cloud technology, becomes compelling for deploying EHR systems because it provides flexible, wide-area mobile access increasingly needed in the modern world. However, before cloud-based EHR systems can become a reality, issues of data security, access control, patient privacy, and overall performance of the whole system must be addressed.

Migrating of EHR to the cloud may represent a great step in the digitalization of medical data. Collaborative model of EHR, like the model described in this paper, will increase the access to the personal and medical data of patients. Some advantages like scalability, costs, and involving the patient as an active part of the health information management process may represent a step forward in healthcare improvement.

Our proposed model of EHR provides integration of all medical data for citizens collected in health care facilities and the administrative data in the HIF. All data from EHR will be enroll in a central location which would be available to all health care workers and institutions according to their access privileges. That integration of health data for citizens in one place provides an opportunity for greater availability and accuracy of data, increased use of the same in various analysis and creating healthcare policies.

Cloud computing technology provides scalability, flexibility and availability of data at any time and from any place. Development of telecommunication services in recent years, allow access to the cloud services from anywhere in the country. All of these previously mentioned advantages of Cloud computing technology and many others are the reason why we propose collaborative model of EHR based on cloud-based technology.

There are many challenges fostering the new collaborative model of cloud computing in healthcare. Several requirements and conditions must be taken into account when sensitive medical and private administrative data are migrating to the cloud based EHR. In order to make this process easier, healthcare providers, or healthcare authorities, must inform their patients of the change and how their data will be managed and stored. The strategic planning is necessary to examine staffing, budget, organizational culture, technologies in the health sector, and government regulations that may affect it, assess its capabilities to achieve the goal, and identify strategies designed to move forward.

Our proposed collaborative model of EHR will provide greater collaboration and exchange data in real time not only between the two major IT systems but also between 
all participants in Macedonian healthcare system by using bigger data protection and digital certificates for authentication and digital signature. In the current situation of the Macedonian health information system, utilization of digital certificates is by one side (of the HIF's side) while the new model offers the possibility of their use in the whole.

Our model of EHR offers the possibility of using digital certificates stored on smart cards, tokens or integration with server-based systems for digital signing. This possibility of integration with server-based systems for authentication and digital signatures makes our model more flexible in the use of new technologies and smart devices (smartphones, tablets). In situation of using digital signatures and authentication, this makes our model hardware independent and independent from additional desktop software which are mandatory when using digital certificates from smart cards.

\section{Conclusion}

Cloud computing technology may widely improve the access to healthcare information in the healthcare system. This technology can provide collaboration between healthcare providers, healthcare staff and citizens. Also, cloud computing technology can provide control the availability of physicians, medical specialist and their service at different times and in different cases.

Using of cloud computing and international healthcare standard in the EHR design, enables integration among many healthcare organizations. This technology reduces complex business processes by automating the manual processes.

Our collaborative model of EHR impact on improving the quality of healthcare services and strengthening the users' role in managing their own healthcare. The use of collaborative EHR system will enable fast data sharing, data analysis tools. All this can speed up the researches for improvement of health care quality. Our proposed model is a conceptual model of collaborative cloud-based model of EHR which expected in future to be implemented.

\section{References}

1. Hayrinen, K., Saranto, K., Nykanen, P.: Definition, structure, content, use and impacts of electronic health records: A review of the research literature. International journal of medical informatics, Vol. 77, 291-304. (2008)

2. Walji, M.F., Kalenderian, E., Piotrowski, M., Tran, D., Kookal, K.K., Tokede, O., et al.: Are three methods better than one? A comparative assessment of usability evaluation methods in an EHR. International journal of medical informatics. Vol. 83, No. 5, 361-368. (2014)

3. Bélanger. E., Bartlett, G., Dawes, M., Rodríguez, C., Gidoni, I.H.: Examining the evidence of the impact of health information technology in primary care: An argument for participatory research with health professionals and patients. International journal of medical informatics. Vol. 81, 654-662. (2012)

4. 4. ISO/DTR 20514:2005, Health Informatics-Electronic Health Record- Definition, Scope, and Context. [Online]. Available at: http://www.iso.org/iso/. (current August 2015)

5. Gavrilov, G., Davcev, D.: Concept of Healthcare Information System in MacedoniaElectronic Health Card System. In Kocarev L, (eds.): ICT Innovations 2011 Web Proceedings ISSN 1857-7288, Skopje, 39-49. (2011) 
6. Ministry of Health, Strategy for the Development of Macedonian Integrated Health Information System. [Online]. Available from: http://zdravstvo.gov.mk/wpcontent/uploads/2015/06/IT_strategija_IZIS.pdf. (current September 2015).

7. Cresswell, K. M., Worth, A., Sheikh, A.: Integration of a nationally procured electronic health record system into user work practices. Medical Informatics and Decision Making. Vol. 12, 15-25. (2012)

8. Law for Healthcare. Official Gazette of the Republic of Macedonia. No. 10/2013, 87/2013, $132 / 2013$, and $164 / 2013$.

9. Law on Health Insurance. Official Gazette of the Republic of Macedonia. No. 16/2013 and $87 / 2013$.

10. Gavrilov, G., Trajkovik, V.: Security and Privacy Issues and Requirements for Healthcare Cloud Computing. In Markovski, S: (eds). ICT Innovations 2012 Web Proceedings ISSN 1857-7288, 143-152. (2012)

11. Health Insurance Fund of Macedonia, Annual Report 2014. [Online]. Available at: http://www.fzo.org.mk/WBStorage/Files/Godisen\%20izvestaj\%202014\%20KONECEN.pdf. (current November 2015)

12. Health Insurance Fund of Macedonia, Technical documentation of e-services. [Online] Available at: www.fzo.org.mk. (current September 2015)

13. Doko, E., Jakimoski, K.: Proposed Module for Gynecological Integration - Preventive Measures in the Electronic Health in Republic of Macedonia. International Journal of BioScience and Bio- Technology, Vol. 7, No. 1, 81-92. (2015)

14. Velinov, G., Jakimovski, B., Lesovski, D., et al.: EHR System Moj Termin: Implementation and Initial Data Analysis. In: Cornet et al. (eds): Digital Healthcare Empowering Europeans. European Federation for Medical Informatics (EFMI), 872-876. (2015)

15. El-Sappagh, S.H., El-Masri, S., Riad, A.M. et al.: Electronic Health Record Data Model Optimized for Knowledge Discovery. International Journal of Computer Science, Vol. 9, No. 5, 329-338. (2012)

16. HL7 Inc. (2014) ISO HL7 21731 'Health informatics - HL7 version 3 Reference Information Model' (RIM). [Online]. Available at: http://www.iso.org/iso/iso_catalogue/catalogue_tc/catalogue_detail.htm?csnumber=61454 , (current July 2015)

17. ISO 13606-1. (2010) Health informatics - Electronic health record communication - Part 1: Reference model. [Online] Available at http://www.iso.org/iso/catalogue_detail.htm?csnumber=40784 , (current July 2015)

18. Beale, T.: The open EHR Archetype Model (AOM) version 1.0.1 (2007). [Online]. Available at: http://www.openehr.org/releases/1.0.1/architecture/am/aom.pdf, (current June 2015).

19. Lopez, D.M., Blobel, B.: Enhanced Semantic Interoperability by Profiling Health Informatics Standards. Methods of Information in Medicine, Vol. 48, No. 2, 170-177. (2009)

20. Cohen, R., Elhadad, M., Elhadad, N.: Redundancy in electronic health record corpora: analysis, impact on text mining performance and mitigation strategies. BMC Bioinformatics, Vol. 14, 10. (2013)

21. Metzger, M.H., Durand, T., Lallich, S., Salamon, R., Castets, P.: The use of regional platforms for managing electronic health records for the production of regional public health indicators in France. BMC Medical Informatics and Decision Making, Vol. 12, 28. (2012)

22. McGinn, C.A., Gagnon, M.P., Shaw, N., Sicotte, C., Mathieu, L., Leduc, Y., et al.: Users' perspectives of key factors to implementing electronic health records in Canada: a Delphi study. BMC Medical Informatics and Decision Making, Vol. 12, 105-115. (2012)

23. Lomotey, R.K., Deters, R.: Efficient consumption of the electronic health record in mHealth. Network Modeling Analysis in Health Informatics and Bioinformatics, Vol. 3, 51-61. (2014)

24. Christine, P. S.: A Glimpse at EHR Implementation Around the World: The Lessons the US Can Learn. The Health Institute for E-Health Policy. (May 2014) 
25. Milieu Ltd and Time.lex, Overview of the national laws on electronic health records in the EU Member States, National Report for Lithuania. [Online]. Available at: https://ec.europa.eu/health/ehealth/docs/laws_lithuania_en.pdf. (current November 2016) 